\title{
Reconfigurable Parralel Multiple in the Galois Fields in Combination Logic
}

\author{
Timur A. Zubov, \\ Vitaly V. Sukhotin, Anton V. Khnykin*, \\ Andrey V. Mishurov and Alexander A. Gorchakovsky \\ Siberian Federal University \\ 79 Svobodny, Krasnoyarsk, 660041, Russia
}

Received 12.06.2019, received in revised form 11.08.2019, accepted 17.09.2019

The concept of "multiplier" in Galois fields, which are widely used in cryptography and noiseresistant coding, is considered. The architecture of a parallel multiplier for the Galois fields is analyzed. Reconfigurable multiplier is constructed. It is shown that the use of this type of multiplier will significantly reduce the number of logic gates.

Keywords: multiplier, Galois fields, combinational logic, reconfigurable module, Bose Chaudhuri Hocquenghem Codes.

\section{Реконфигурируемый параллельный умножитель в конечных полях Галуа на комбинационной логике}

\author{
Т.А. Зубов, В.В. Сухотин, А.В. Хныкин, \\ А.В. Мишуров, А.А. Горчаковский \\ Сибирский федеральный университет \\ Россия, 660041, Красноярск, пр. Свободньй, 79
}

Рассмотрено понятие «умножитель» в конечных полях Галуа, имеющих широкое применение 8 криптографии и помехоустойчивом кодировании. Проанализирована архитектура параллельного умножителя над полями Галуа. Проведено построение реконфигурируемого умножителя. Показано, что применение данного типа умножителя позволит значительно сократить число применяемых логических вентилей.

Ключевые слова: умножитель, поля Галуа, комбинационная логика, реконфигурируемыймодуль, коды Боуза-Чоудхури-Хоквингема.

(c) Siberian Federal University. All rights reserved

This work is licensed under a Creative Commons Attribution-NonCommercial 4.0 International License (CC BY-NC 4.0).

* Corresponding author E-mail address: akhnykin@sfu-kras.ru 


\section{Введение}

В нашу жизнь очень глубоко проникли цифровые технологии - от гаджетов до систем передачи информации. Очень большое внимание уделяется развитию стандартов передачи информации DVB-S2 и DVB-S2X в части обеспечения помехоустойчивости и оптимизации структур умножителей, входящих в состав кодеров/декодеров. Применение достаточно сложных кодов приводит к увеличению количества структур умножителей над несколькими полями Галуа, увеличению времени обработки информации и неэффективного использования программируемых логических интегральных схем (ПЛИС). Для того чтобы свести к минимуму или исключить указанные недостатки, требуется создать реконфигурируемый умножитель с минимальным количеством вентилей. Рассмотрим возможность построения данного умножителя и сделаем соответствующие выводы.

\section{1. Что такое умножитель}

Арифметика в полях Галуа широко используется в криптографии и помехоустойчивом кодировании, в частности в кодах Боуза-Чоудхури-Хоквингема (БЧХ), относящихся к семейству циклических кодов [1].

Умножение двух величин в конечном двоичном поле Галуа степени $m \operatorname{GF}\left(2^{m}\right)$ представляется следующим образом [2]:

$$
C(x)=A(x) B(x) \bmod P(x),
$$

где $A(x)=a_{m-1} x^{m-1}+a_{m-1} x^{m-2}+\ldots+a_{1} x+a_{0}=\sum_{i=0}^{m-1} a_{i} x^{i}-$ полином степени $m-1$,

$$
\begin{aligned}
& B(x)=b_{m-1} x^{m-1}+b_{m-1} x^{m-2}+\ldots+b_{1} x+b_{0}=\sum_{i=0}^{m-1} b_{i} x^{i}-\text { полином степени } m-1, \\
& P(x)=p_{m} x^{m}+p_{m-1} x^{m-1}+\ldots+p_{1} x+p_{0}=p_{m} x^{m}+\sum_{i=0}^{m-1} p_{i} x^{i}-\text { неприводимый полином степени } m,
\end{aligned}
$$

порождающий поле $\mathrm{GF}\left(2^{m}\right)$.

В конечном поле имеется примитивный элемент $\alpha \in G F\left(2^{m}\right)$, степени которого порождают ненулевые элементы поля, т.е. $\beta=\alpha^{i} \in G F\left(2^{m}\right), 0 \leq i \leq 2^{m}-2$. Элемент $\alpha$ является корнем неприводимого двоичного полинома $P(x), P(\alpha)=0$ [2, с. 81]. Умножение двух величин (1) представляется следующим образом:

где

$$
S=A(\alpha) B(\alpha)=\left(\sum_{i=0}^{m-1} a_{i} \alpha^{i}\right)\left(\sum_{j=0}^{m-1} b_{j} \alpha^{j}\right)=\sum_{k=0}^{2 m-1} s_{k} \alpha^{k},
$$

$$
s_{k}=\sum_{i+i=k} a_{i} b_{j}, 0 \leq i, j \leq m-1,0 \leq k \leq 2 m-1 .
$$

Полная операция умножения (1) двух величин в конечном двоичном поле Галуа такова [3]:

$$
C \triangleq \sum_{i=0}^{m-1} c_{i} a^{i} \equiv \operatorname{Smod} P(a) .
$$

\section{2. Архитектура параллельного умножителя над полями Галуа}

Для аппаратной реализации выражения (1) и (2) представляются в матричной форме [4]:

$$
-803-
$$




$$
C=d+Q^{T} e,
$$

где $\mathrm{Q}$ - двоичная матрица остатков:

$$
\begin{aligned}
& Q=\left[\begin{array}{c}
\alpha^{m} \bmod P(\alpha) \\
\alpha^{m+1} \bmod P(\alpha) \\
\vdots \\
\alpha^{2 m-1} \bmod P(\alpha)
\end{array}\right], \\
& d=L B, \\
& e=U B,
\end{aligned}
$$

$L$ и $U$ - матрицы Теплица:

$$
L \triangleq\left[\begin{array}{ccccc}
a_{0} & 0 & 0 & \ldots & 0 \\
a_{1} & a_{1} & 0 & \ldots & 0 \\
\vdots & \vdots & \ddots & \ddots & \vdots \\
a_{m-2} & a_{m-3} & \ldots & a_{0} & 0 \\
a_{m-1} & a_{m-2} & \ldots & a_{1} & a_{0}
\end{array}\right], U \triangleq\left[\begin{array}{ccccc}
0 & a_{m-1} & a_{m-2} & \ldots & a_{1} \\
0 & 0 & a_{m-1} & \ldots & a_{2} \\
\vdots & \vdots & \ddots & \ddots & \vdots \\
0 & 0 & \ldots & 0 & a_{m-1}
\end{array}\right] .
$$

Архитектура low complexity bit parallel (LCBP) умножителя над полем Галуа, основанного на формуле (8) и архитектуре Мастравито [5], показана на рис. 1 [4].

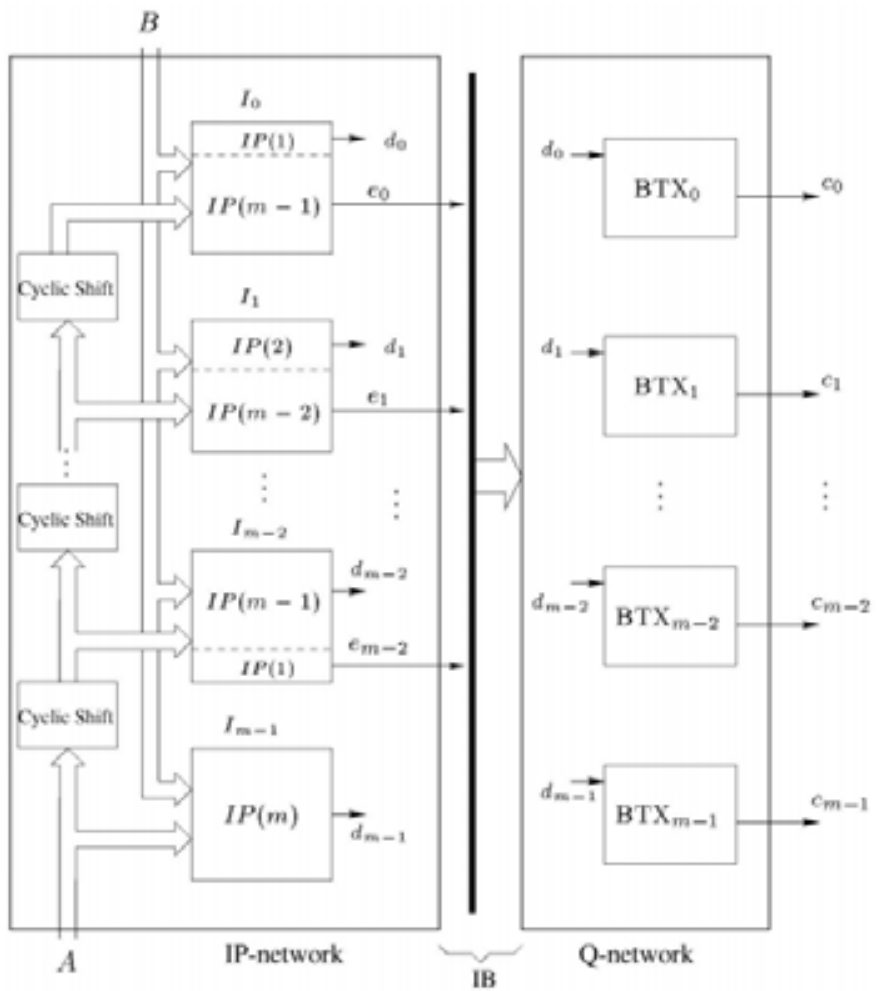

Рис. 1. Архитектура умножителя LCBP над полем $\mathrm{GF}\left(2^{m}\right)$

Fig. 1. The architecture of the LCBP multiplier for the $\operatorname{GF}\left(2^{m}\right)$ 
Архитектура состоит из двух частей: $I P$-область и $Q$-область. $I P$-область состоит из $m$ $I$-блоков, вычисляющих вектора $d$ и $e$ по выражениям (6) и (7). $Q$-область состоит из $m$ блоков $\mathrm{BTX}_{0 . \ldots m-1}$, содержащих исключающие сумматоры XOR. Количество сумматоров BTX $(i$, $0 \leq i \leq m-1)$ определяется количеством единиц в столбцах матрицы $Q$. Блок «Cyclic Shift» сдвигает старший разряд вектора на нулевую позицию, как показано на рис. 2.

Так, например, архитектура умножителя над полем $\mathrm{GF}\left(2^{4}\right)$, генерируемого полиномом $p(x)=x^{4}+x+1$, показана на рис. 3 [4].

Вычисление векторов $d$ и $е$ осуществляется при помощи полиномиального умножения в $I P$-области умножителя над полем $\mathrm{GF}\left(2^{4}\right)$ и выглядит следующим образом (рис. 4) [6].

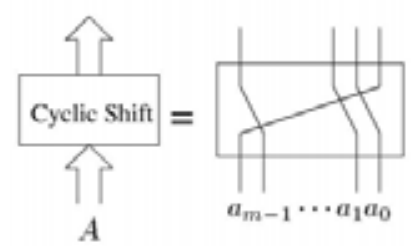

Рис. 2. Устройство блока «Cyclic Shift»

Fig. 2. Block «Cyclic Shift»

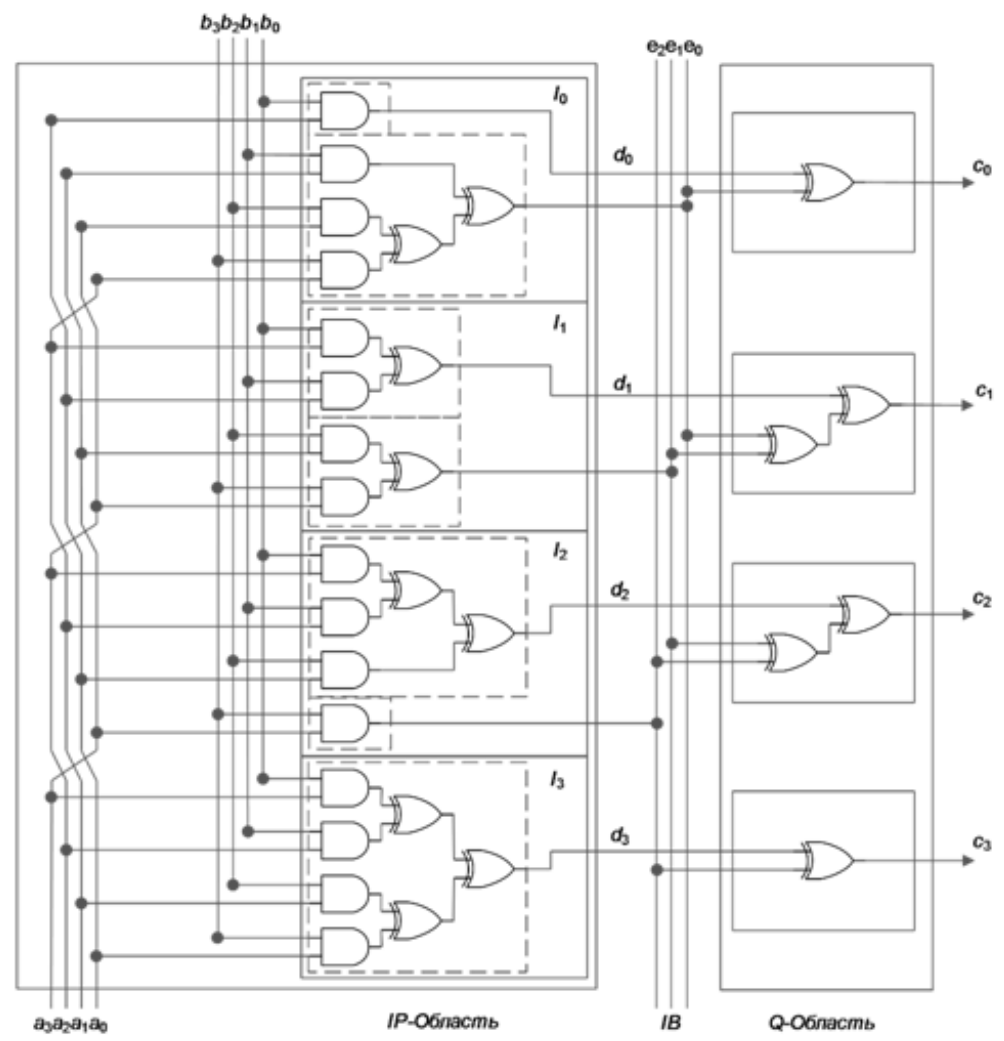

Рис. 3. Архитектура умножителя над полем $\mathrm{GF}\left(2^{4}\right)$

Fig. 3. Multiplier architecture for $\mathrm{GF}\left(2^{4}\right)$ 


\begin{tabular}{|c|c|c|c|c|c|c|c|}
\hline & $\alpha^{0}$ & $\alpha$ & $\alpha^{2}$ & $\alpha^{3}$ & $\alpha^{4}$ & $\alpha^{5}$ & $\alpha^{6}$ \\
\hline \multirow{2}{*}{$\otimes$} & $\mathrm{A}_{0}$ & $\mathrm{~A}_{\mathrm{l}}$ & $\mathrm{A}_{2}$ & $\mathrm{~A}_{3}$ & & & \\
\hline & $\mathrm{B}_{0}$ & $\mathrm{~B}_{1}$ & $\mathrm{~B}_{2}$ & $\mathrm{~B}_{3}$ & & & \\
\hline \multirow{4}{*}{$\begin{array}{l}\text { (1) } \\
\text { (1) } \\
\text { (1) }\end{array}$} & $\mathrm{A}_{0} \mathrm{~B}_{0}$ & $\mathrm{~A}_{1} \mathrm{~B}_{0}$ & $\mathrm{~A}_{2} \mathrm{~B}_{0}$ & $\mathrm{~A}_{3} \mathrm{~B}_{0}$ & & & \\
\hline & & $\mathrm{A}_{0} \mathrm{~B}_{1}$ & $\mathrm{~A}_{1} \mathrm{~B}_{1}$ & $\mathrm{~A}_{2} \mathrm{~B}_{1}$ & $\mathrm{~A}_{3} \mathrm{~B}_{1}$ & & \\
\hline & & & $\mathrm{A}_{0} \mathrm{~B}_{2}$ & $\mathrm{~A}_{1} \mathrm{~B}_{2}$ & $\mathrm{~A}_{2} \mathrm{~B}_{2}$ & $\mathrm{~A}_{3} \mathrm{~B}_{2}$ & \\
\hline & & & & $\mathrm{A}_{0} \mathrm{~B}_{3}$ & $\mathrm{~A}_{1} \mathrm{~B}_{3}$ & $\mathrm{~A}_{2} \mathrm{~B}_{3}$ & $\mathrm{~A}_{3} \mathrm{~B}_{3}$ \\
\hline
\end{tabular}

Рис. 4. Вычисление векторов $d$ и $е$ для поля $\mathrm{GF}\left(2^{4}\right)$

Fig. 4. Calculation of vectors $d$ and $e$ for the field $\mathrm{GF}\left(2^{4}\right)$

Количество блоков $\mathrm{XOR}$ и входные сигналы определяются матрицей $Q$, которая рассчитывается по формуле (6). Для умножителя над полем GF(24) матрица будет выглядеть так:

$$
\begin{aligned}
& \begin{array}{llll}
d_{0} & d_{1} & d_{2} & d_{3}
\end{array} \\
& Q=\left[\begin{array}{llll}
1 & 1 & 0 & 0 \\
0 & 1 & 1 & 0 \\
0 & 0 & 1 & 1
\end{array}\right] \begin{array}{l}
e_{0} \\
e_{1} \\
e_{2}
\end{array} .
\end{aligned}
$$

Тогда величина $C$ (5) будет вычислена следующим образом:

$$
\left[\begin{array}{c}
c_{0} \\
c_{1} \\
c_{2} \\
c_{3}
\end{array}\right]=\left[\begin{array}{c}
d_{0} \oplus e_{0} \\
d_{1} \oplus e_{0} \oplus e_{1} \\
d_{2} \oplus e_{1} \oplus e_{2} \\
d_{3} \oplus e_{2}
\end{array}\right] .
$$

\section{3. Построение параллельного реконфигурируемого умножителя}

На практике не всегда используется умножение над одним полем Галуа, в частности, если рассматривать декодирования БЧХ(ВCH) в стандарте DVB-S2X [7], то здесь используется умножение с тремя разными полями Галуа: $\mathrm{GF}\left(2^{16}\right)$ (нормальный FECFRAME, $n=64800$ ), $\mathrm{GF}\left(2^{15}\right)$ (средний FECFRAME, $n=32400$ ) и $\mathrm{GF}\left(2^{14}\right)$ (короткий FECFRAME, $n=16200$ ), что требует наличие трех разных умножителей над тремя полями Галуа. Реализация каждого умножителя на основе структуры, показанной на рис. 1 , достаточно затратная задача с точки зрения ресурсов. Только для $I P$-области потребуется $m^{2}$ логических блоков AND и $(m-1)^{2}$ логических блоков XOR, а для $Q$-области количество логических блоков XOR определяется количеством единиц в матрице $Q$. Количество вентилей для всех трех полей Галуа сведены в табл.

Как видно из данных таблицы, большая часть вентилей сосредоточена в IP-области. Чтобы построить реконфигурируемый умножитель, необходимо сформировать единую структуру для всех трех полей.

Перед тем как перейти к построению реконфигурируемого умножителя для стандарта DVB-S2X, рассмотрим возможность построения реконфигурируемого умножителя на двух элементарных полях. За основу возьмем архитектуру на рис. 3 с порождающим поле $\mathrm{GF}\left(2^{4}\right)$ 
Таблица. Количество вентилей в умножителях над полями Галуа

Table. The number of logic gates in multipliers for the Galois fields

\begin{tabular}{|c|c|c|c|c|}
\hline \multirow{2}{*}{ Поле } & \multirow{2}{*}{$\begin{array}{c}\text { Порождающий } \\
\text { полином } P(x)\end{array}$} & AND, шт. & XOR, шт. & ХР-Область \\
\cline { 3 - 5 } & $1+x^{2}+x^{3}+x^{5}+x^{16}$ & 256 & 225 & 71 \\
\hline $\mathrm{GF}\left(2^{16}\right)$ & $1+x^{2}+x^{3}+x^{5}+x^{15}$ & 225 & 196 & 67 \\
\hline $\mathrm{GF}\left(2^{15}\right)$ & $1+x+x^{3}+x^{5}+x^{14}$ & 196 & 169 & 62 \\
\hline $\mathrm{GF}\left(2^{14}\right)$ & &
\end{tabular}

полиномом $p(x)=x^{4}+x+1$ и к нему добавим $p(x)=x^{3}+x+1$, генерирующим $\mathrm{GF}\left(2^{3}\right)$. Выбор данных полиномов связан с тем, что младшие члены полиномов близки или одинаковы, такую же схожесть имеют полиномы, приведенные в табл. Полиномиальное умножение для $\mathrm{GF}\left(2^{3}\right)$ в $I P$-области для вычисления векторов $d$ и $e$ показано на рис. 5 .

Матрица остатков для поля $\mathrm{GF}\left(2^{3}\right)$, рассчитанная по формуле (6), выглядит так:

$$
\begin{aligned}
& \begin{array}{llll}
d_{0} & d_{1} & d_{2}
\end{array} \\
& Q=\left[\begin{array}{lll}
1 & 1 & 0 \\
0 & 1 & 1
\end{array}\right] \begin{array}{l}
e_{0} . \\
e_{1}
\end{array}
\end{aligned}
$$

Величина $C$ (5) будет вычислена следующим образом:

$$
\left[\begin{array}{c}
c_{0} \\
c_{1} \\
c_{2}
\end{array}\right]=\left[\begin{array}{c}
d_{0} \oplus e_{0} \\
d_{1} \oplus e_{0} \oplus e_{1} \\
d_{2} \oplus e_{1}
\end{array}\right] .
$$

Сравнив полиномиальное умножение для $\mathrm{GF}\left(2^{3}\right)$ и $\mathrm{GF}\left(2^{4}\right)$ на рис. 3 и 5 , можно увидеть, что полиномиальное умножение для $\mathrm{GF}\left(2^{3}\right)$ можно осуществить в схеме, предназначенной для $\mathrm{GF}\left(2^{4}\right)$ благодаря тому, что свертка - линейная операция. Прибегнув к обнулению старших разрядов $a$ и $b$ на входе и к линейному преобразованию элементов векторов $d$ и $e$ на выходе, можно

\begin{tabular}{|c|c|c|c|c|c|}
\hline \multirow{3}{*}{$\otimes$} & $\alpha^{0}$ & $\alpha$ & $\alpha^{2}$ & \multirow[t]{3}{*}{$\alpha^{3}$} & \multirow[t]{3}{*}{$\alpha^{4}$} \\
\hline & $\mathrm{A}_{0}$ & $\mathrm{~A}_{1}$ & $\mathrm{~A}_{2}$ & & \\
\hline & $\mathrm{B}_{0}$ & $\mathrm{~B}_{1}$ & $\mathrm{~B}_{2}$ & & \\
\hline \multirow{3}{*}{$\begin{array}{l}\text { (1) } \\
\text { (1) }\end{array}$} & $\mathrm{A}_{0} \mathrm{~B}_{0}$ & $\mathrm{~A}_{1} \mathrm{~B}_{0}$ & $\mathrm{~A}_{2} \mathrm{~B}_{0}$ & & \\
\hline & & $\mathrm{A}_{0} \mathrm{~B}_{\mathrm{I}}$ & $\mathrm{A}_{1} \mathrm{~B}_{1}$ & $\mathrm{~A}_{2} \mathrm{~B}_{1}$ & \\
\hline & & & $\mathrm{A}_{0} \mathrm{~B}_{2}$ & $\mathrm{~A}_{1} \mathrm{~B}_{2}$ & $\mathrm{~A}_{2} \mathrm{~B}_{2}$ \\
\hline
\end{tabular}
показать умножение для $\mathrm{GF}\left(2^{3}\right)$ в схеме $\mathrm{GF}\left(2^{4}\right)$, что отображено на рис. 6.

Вектора $d$ ' и $e^{\prime}$ - преобразованные вектора $d$ и $e$, адаптированные для $\operatorname{GF}\left(2^{3}\right)$.

Рис. 5. Вычисление векторов $d$ и $е$ для поля $\mathrm{GF}\left(2^{3}\right)$

Fig. 5. Calculation of vectors $d$ and $e$ for the $\mathrm{GF}\left(2^{3}\right)$ 


\begin{tabular}{|c|c|c|c|c|c|c|c|}
\hline \multirow{3}{*}{8} & $\alpha^{\mathrm{C}}$ & $\alpha$ & $\alpha^{2}$ & $\alpha^{3}$ & $\alpha^{4}$ & $\alpha^{5}$ & $\alpha^{6}$ \\
\hline & $\mathrm{Ac}_{\mathrm{c}}$ & $A_{1}$ & $\mathrm{~A}_{2}$ & 0 & & & \\
\hline & $\mathrm{B}_{\mathrm{c}}$ & $\mathrm{B}_{1}$ & $\mathrm{~B}_{2}$ & 0 & & & \\
\hline \multirow{4}{*}{$\begin{array}{l}\theta \\
\theta\end{array}$} & $\mathrm{A}_{0} \mathrm{~B}_{0}$ & $A_{1} B_{0}$ & $\mathrm{~A}_{2} \mathrm{~B}_{0}$ & 0 & & & \\
\hline & & \multirow{3}{*}{$\mathrm{A}_{0} \mathrm{~B}_{1}$} & $\mathrm{~A}_{1} \mathrm{~B}_{1}$ & $\mathrm{~A}_{2} \mathrm{~B}_{1}$ & 0 & & \\
\hline & & & $\mathrm{A}_{0} \mathrm{~B}_{2}$ & $\mathrm{~A}_{1} \mathrm{~B}_{2}$ & $\mathrm{~A}_{2} \mathrm{~B}_{2}$ & 0 & \\
\hline & & & & 0 & 0 & 0 & 0 \\
\hline$G F\left(2^{4}\right)$ & $d_{0}$ & $d_{1}$ & $d_{2}$ & $d_{3}$ & $e_{0}$ & $e_{1}$ & $e_{2}$ \\
\hline \multirow{2}{*}{$G F\left(2^{3}\right)$} & $d_{0}$ & $d_{1}$ & $d_{2}$ & $e_{0}$ & $e_{1}$ & 0 & 0 \\
\hline & $d_{0}^{\prime}$ & $d_{1}^{\prime}$ & $d_{2}^{\prime}$ & $e_{0}^{\prime}$ & $e_{1}^{\prime}$ & $d^{\prime}{ }_{3}$ & $e_{2}^{\prime}$ \\
\hline
\end{tabular}

Рис. 6. Вычисление векторов $d$ и е поля $\mathrm{GF}\left(2^{3}\right)$ в схеме, предназначенной для $\mathrm{GF}\left(2^{4}\right)$

Fig. 6. Calculation of vectors $d$ and $e$ of the $\mathrm{GF}\left(2^{3}\right)$ in the scheme for $\mathrm{GF}\left(2^{4}\right)$

Рассмотрев матрицы (10) и (11), можно увидеть, что матрица (11) является подматрицей (10), которая выделена в выражении:

$$
\begin{aligned}
& \begin{array}{llll}
d_{0}^{\prime} & d_{1}^{\prime} & d^{\prime}{ }_{2} & 0
\end{array}
\end{aligned}
$$

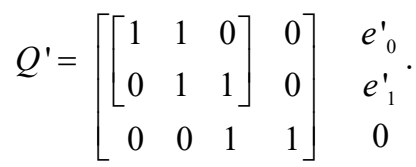

Величина $C$ будет следующей:

$$
\left[\begin{array}{c}
c_{0}^{\prime} \\
c_{1}^{\prime} \\
c_{1}^{\prime}{ }_{2} \\
c_{3}^{\prime}
\end{array}\right]=\left[\begin{array}{c}
d_{0}^{\prime} \oplus e_{0}^{\prime} \\
d_{1}^{\prime} \oplus e_{0}^{\prime} \oplus e_{1}^{\prime} \\
d_{2}^{\prime} \oplus e_{1}^{\prime} \oplus e_{2}^{\prime} \\
d_{3}^{\prime} \oplus e_{2}^{\prime}
\end{array}\right]=\left[\begin{array}{c}
d_{0}^{\prime} \oplus e_{0}^{\prime} \\
d_{1}^{\prime} \oplus e_{0}^{\prime} \oplus e_{1}^{\prime} \\
d_{2}^{\prime} \oplus e_{1}^{\prime} \oplus 0 \\
0 \oplus 0
\end{array}\right]=\left[\begin{array}{c}
d_{0}^{\prime} \oplus e_{0}^{\prime} \\
d_{1}^{\prime} \oplus e_{0}^{\prime} \oplus e_{1}^{\prime} \\
d_{2}^{\prime} \oplus e_{1}^{\prime} \\
0
\end{array}\right]=\left[\begin{array}{c}
C_{G F\left(2^{3}\right)} \\
0
\end{array}\right] .
$$

Таким образом, блоки $\mathrm{BTX}_{i}(0 \leq i \leq 3)$ остаются без внесения изменений и реконфигурируемая архитектура будет выглядеть, как это показано на рис. 7.

На рис. 7 видно, что количество умножителей AND и исключающих сумматоров в IPобласти, ограничиваясь старшим полем, до значений $4^{2}$ и $3^{2}$ соответственно вместо $4^{2}+3^{2}$ и $3^{2}+2^{2}$ для двух отдельных архитектур, уменьшено.

\section{Заключение}

Реконфигурируемый умножитель над полями Галуа сформирован. Построенная реконфигурируемая архитектура параллельного умножителя над двумя полями Галуа $\mathrm{GF}\left(2^{4}\right)$ и $\mathrm{GF}\left(2^{3}\right)$ позволяет сократить количество умножителей AND и исключающих сумматоров в $I P$-области, ограничиваясь старшим полем, до значений $4^{2}$ и $3^{2}$ соответственно вместо $4^{2}+3^{2}$ и $3^{2}+2^{2}$ для двух отдельных архитектур. Также перестановкой элементов векторов $d$ и $e$ и сопоставлением расположения ненулевых элементов матриц остатков $Q$ можно минимизировать количество исключающих сумматоров XOR в $Q$-области. Предложенный подход к построению реконфигурируе- 


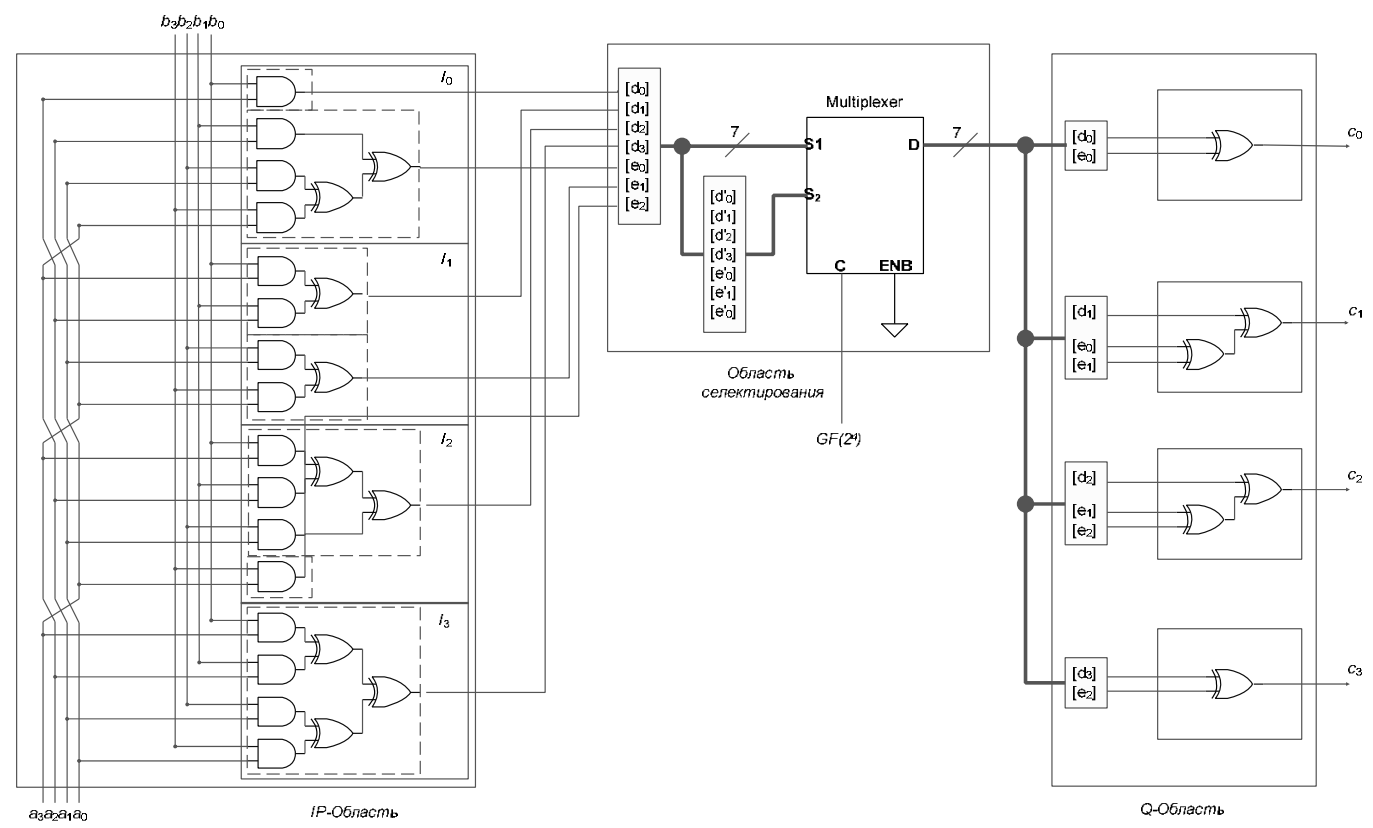

Рис. 7. Реконфигурируемая архитектура умножителя над полями $\operatorname{GF}\left(2^{4}\right)$ и $\operatorname{GF}\left(2^{3}\right)$

Fig. 7. Reconfigurable multiplier architecture for $\operatorname{GF}\left(2^{4}\right)$ and $\operatorname{GF}\left(2^{3}\right)$

мого умножителя, исходя из принятых условий, возможно распространить для формирования такого в стандарте DVB-S2X.

\section{Список литературы}

[1] Сагалович Ю.Л. Введение в алгебраические коды. М.: МФТИ, 2007. 262 с. [Sagalovich Yu.L. Introduction to algebraic codes, Moscow, MIPT, 2007, 262 p. (in Russian)]

[2] Mathew J., Jabir A.M., Rahaman H., Pradhan D.K. Single error correctable bit parallel multipliers over GF $\left(2^{\mathrm{m}}\right)$, IET Computers \& Digital Techniques, 2009, 3(3), 281-288.

[3] Морелос-Сарагоса Р. Искусство помехоустойчивого кодирования. Методы, алгоритмы, применение. М.: Техносфера, 2005. 320 c. [Morelos-Zaragoza R. The art of noise-tolerant coding. Methods, algorithms, application, Moscow, Technosphere, 2005, 320 p. (in Russian)]

[4] Reyhani-Masoleh A., Anwar Hasan M. Low Complexity Bit Parallel Architectures for Polynomial Basis Multiplication over GF( $\left.2^{\mathrm{m}}\right)$, IEEE Transactions on Computers, 2004, 53(8), 945959.

[5] Halbutogullari A., Koc C. Mastrovito Multiplier for General Inrreducible Polynominals, IEEE Transactions on Computers, 2000, 49(5), 503-518.

[6] Lee C.Y., Lu E.H. Lee J.Y. Bit-Parallel Systolic Multipliers for GF $\left(2^{\mathrm{m}}\right)$ Fields Defined by AllOne and Equally Spaced Polynomials, IEEE Transactions on Computers, 2001, 50(5), 385-393.

[7] ETSI. Digital video broadcasting (DVB). Second generation framing structure, channel coding and modulation systems for broadcasting, interactive services, news gathering and other broadband satellite applications; Part 2: DVB-S2 Extensions (DVB-S2X): EN 302 307-2 V1.3.1, 2014. 